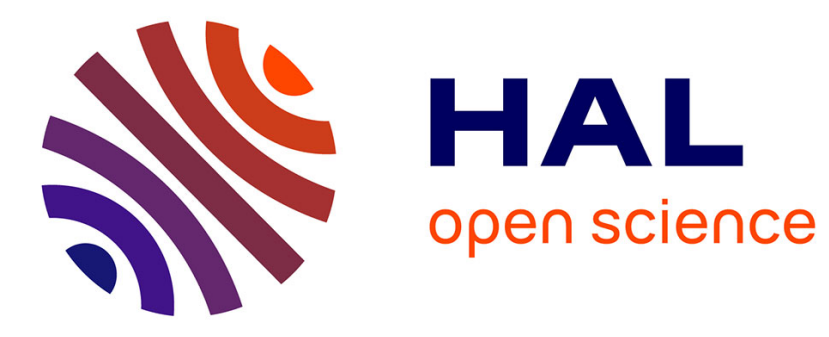

\title{
Quantum Well States for Graphene Spin-Texture Engineering
}

Thomas Vincent, Elena Voloshina, Stéphane Pons, Sabina Simon, Mikhail Fonin, Kangli Wang, Beate Paulus, Dimitri Roditchev, Yuriy Dedkov, Sergio Vlaic

\section{To cite this version:}

Thomas Vincent, Elena Voloshina, Stéphane Pons, Sabina Simon, Mikhail Fonin, et al.. Quantum Well States for Graphene Spin-Texture Engineering. Journal of Physical Chemistry Letters, 2020, 11 (4), pp.1594-1600. 10.1021/acs.jpclett.0c00069 . hal-03035667

\section{HAL Id: hal-03035667 https://hal.science/hal-03035667}

Submitted on 2 Dec 2020

HAL is a multi-disciplinary open access archive for the deposit and dissemination of scientific research documents, whether they are published or not. The documents may come from teaching and research institutions in France or abroad, or from public or private research centers.
L'archive ouverte pluridisciplinaire HAL, est destinée au dépôt et à la diffusion de documents scientifiques de niveau recherche, publiés ou non, émanant des établissements d'enseignement et de recherche français ou étrangers, des laboratoires publics ou privés. 


\section{Quantum Well States for Graphene Spin-Texture Engineering}

Thomas Vincent, ${ }^{\dagger}$ Elena Voloshina, ${ }^{*,+}, \mathbb{\uparrow}, \S$ Stephane Pons, ${ }^{\dagger}$ Sabina Simon, Fonin," Kangli Wang, " Beate Paulus, " Dimitri Roditchev, ${ }^{\dagger, \perp}$ Yuriy Dedkov, ${ }^{*}, \S, \S$ and Sergio Vlaic*,

$\dagger$ Laboratoire de Physique et d'Étude des Matériaux, ESPCI Paris, PSL Research University, CNRS, UMR 8213, Sorbonne Universités, UPMC Univ. Paris 06, 75005 Paris, France

$\ddagger$ Department of Physics, Shanghai University, 99 Shangda Road, 200444 Shanghai, China IInstitut für Chemie und Biochemie, Freie Universität Berlin, Takustrasse 3, 14195 Berlin, Germany

§Institute of Physical and Organic Chemistry, Southern Federal University, 344090 Rostov on Don, Russia

||Department of Physics, University of Konstanz, 78457 Konstanz, Germany $\perp I N S P$, UPMC Paris 6 and CNRS-UMR 7588, 4 place Jussieu, 75252 Paris, France

E-mail: voloshina@shu.edu.cn; dedkov@shu.edu.cn; sergio.vlaic@espci.fr 


\begin{abstract}
The modification of graphene band structure, in particular via induced spin-orbit coupling, is currently a great challenge for the scientific community from both a fundamental and applied point of view. Here, we investigate the modification of the electronic structure of graphene (gr) initially adsorbed on $\operatorname{Ir}(111)$ via intercalation of one monolayer Pd by means of angle-resolved photoelectron spectroscopy and density functional theory. We reveal that for the $\mathrm{gr} / \mathrm{Pd} / \operatorname{Ir}(111)$ intercalated system, a spin splitting of graphene $\pi$ states higher than $200 \mathrm{meV}$ is present near the graphene $K$ point. This spin-separation arises from the hybridization of the graphene valence band states with spin-polarized quantum well states of a single Pd layer on $\operatorname{Ir}(111)$. Our results demonstrate that the proposed approach on the tailoring of the dimensionality of heavy materials interfaced with a graphene layer might lead to a giant spin-orbit splitting of the graphene valence band states.
\end{abstract}

\title{
Graphical TOC Entry
}

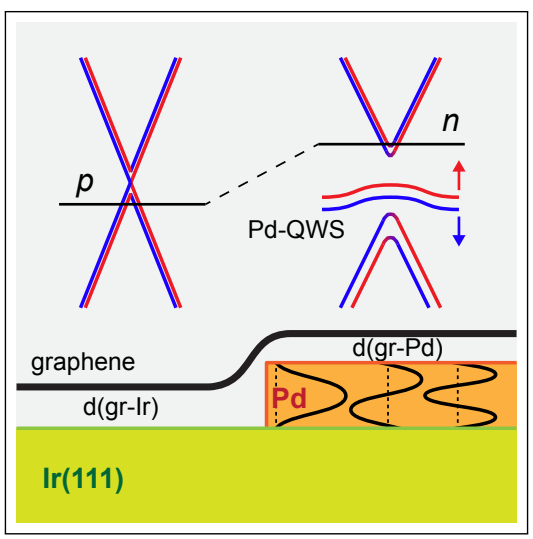


Since the discovery of the exceptional electronic features of graphene (gr) a great challenge for solid state physics and material science has been to expand its functionalities by the introduction of novel properties. ${ }^{\frac{11}{3}}$ Recently, a strong attention has been devoted to the use of graphene for spintronic applications, with the ultimate goal of manipulating the spin by external electric field. ${ }^{223}$ In fact, being made by a light element such as carbon, graphene presents an intrinsic weak spin-orbit interaction, while its enhancement is expected to pave the way to a multitude of exotic phenomena, such as quantum spin Hall effect ${ }^{415}$ and possible applications as spin injectors or spin filters. .617

One of the most used approach to induce or/and enhance the spin-orbit interaction in graphene has been by proximity with metallic compounds. ${ }^{[19}$ In such a case two main mechanisms can be at work: Rashba effect and induced spin-orbit interaction by direct hybridization of the graphene $\pi$ states with the valence band states of the metal. The resulting spin-splitting is expected to present a different configuration depending on the leading mechanism: a constant energy separation as a function of the wave vector in the vicinity of the Dirac point of the graphene $\pi$ states in the case of Rashba splitting, while an energy and momentum dependent splitting for the graphene $\pi$ states, with strong reduction when approaching the Fermi energy $\left(E_{F}\right)$, in the case of direct hybridization of the valence band states of graphene and metal. ${ }^{[20]}$

Several studies have identified a spin separation of graphene bands when graphene is supported on magnetic materials ${ }^{8 / 1221}$ or on heavy metal compounds. $\frac{13 \cdot 19}{19}$ The origin of such spin splitting is not straight-forward. In the case of magnetic substrates, the binding energy variation of the graphene $\pi$ states upon magnetization reversal has been attributed to Rashba effect for graphene on $\mathrm{Ni}, \underline{10}$ while spin splitting of these states was assigned to hybridization between valence band states of graphene and Co. ${ }^{11122}$ In the case of graphene on heavy metals the situation is more complicated. While most of the studies performed by means of spin-resolved photoelectron spectroscopy revealed spin splitting of the order of 
$100 \mathrm{meV}$ in the vicinity of $E_{F}$ and attributed to direct carbon-substrate valence band states hybridization, $\frac{1316] 18}{18}$ such behavior is not reproduced by other experimental techniques such as non spin-resolved angle-resolved photoelectron spectroscopy (ARPES) with high energyand $k$-resolution, even in very high quality samples ${ }^{2224}$ or scanning tunneling microscopy (STM) on very similar systems. ${ }^{25}$ 27 Moreover these studies have been performed on systems where the graphene-substrate interaction is relatively small (i. e. Au, Pt, and Ir). When graphene is lying on such materials the average carbon-substrate distance is expected to be well above $3 \AA$, thus preserving the Dirac cone linear dispersion and preventing strong valence band states hybridization effects. ${ }^{[20}$ It has to be noticed that, so far, the scientific effort has been focused on interfacing graphene with metals which possess strong (e.g., Co and $\mathrm{Ni}$ ) or weak (e.g., Au, Pt, Ir) interaction with carbon atoms.

In this manuscript we present a different approach focusing our attention on graphene placed on Pd. In such a case the gr-Pd interaction lies in between to what is considered strongly (e.g., C-Co interaction: $175 \mathrm{meV} / \mathrm{C}-\mathrm{atom}$ ) and weekly interacting (e.g., C-Pt interaction: $33 \mathrm{meV} / \mathrm{C}$-atom) having an expected interaction of $79 \mathrm{meV} / \mathrm{C}$-atom. ${ }^{28}$ We show, by a combination of ARPES, STM, and density functional theory (DFT), that graphene interfaced with one monolayer (ML) Pd on $\operatorname{Ir}(111)$ presents a giant spin-splitting of the $\pi$ states up to $200 \mathrm{meV}$ in the vicinity of $E_{F}$ and the Dirac point, resulting from the hybridization between the graphene valence band states with the spin-split quantum well states of $\mathrm{Pd} / \operatorname{Ir}(111)$. These results show that our approach of using spin-polarized quantum well states for graphene band engineering might lead to giant spin-splitting in the graphene valence band. Details describing experimental and theoretical approaches used in the present study as well as additional data are presented in the Supporting Information.

The top and side views of the $\mathrm{gr} / \mathrm{Pd} / \operatorname{Ir}(111)$ interface, obtained after structural optimization in DFT calculations, are shown in Fig. $1(\mathrm{a}, \mathrm{b})$ where Pd atoms are placed above $\operatorname{Ir}(111)$ assuming the pseudomorphic growth. Compared to the parent gr/Ir(111) system, 
a)

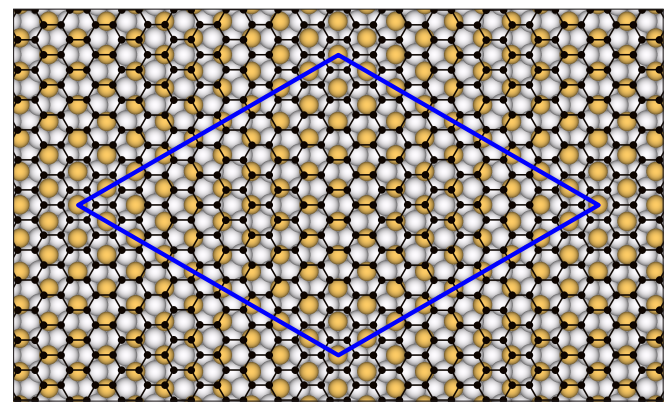

b)

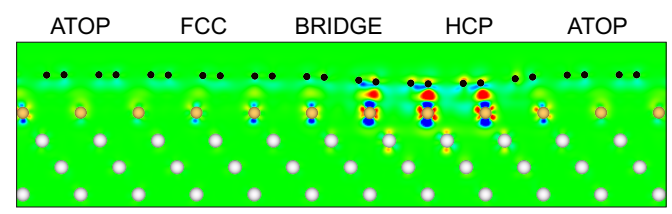

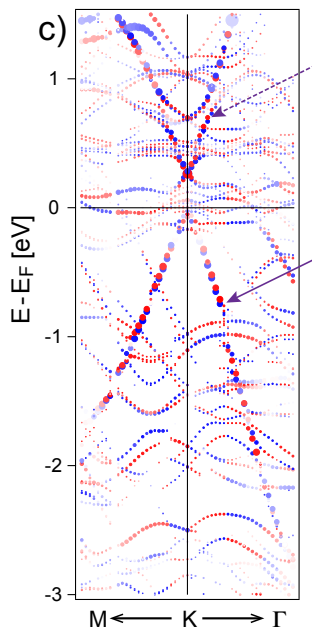
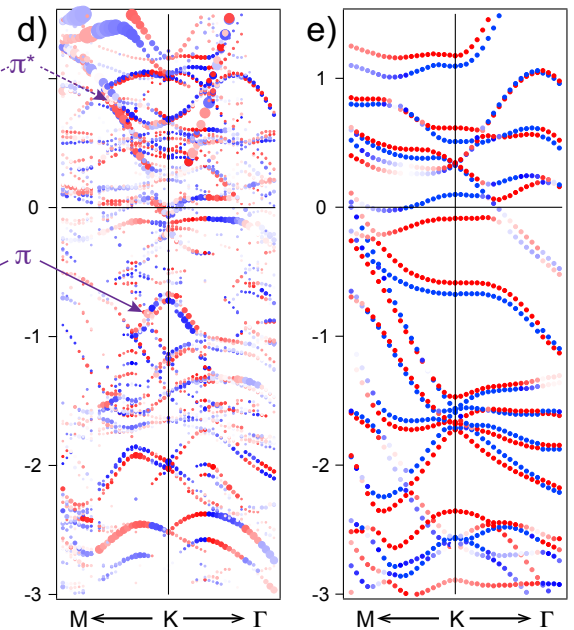

Figure 1: (a) Top view of the crystallographic structure of $\mathrm{gr} / \mathrm{Pd} / \operatorname{Ir}(111)$. Blue rhombus marks the unit cell. (b) Side view of the fully relaxed $\mathrm{gr} / \mathrm{Pd} / \operatorname{Ir}(111)$ interface is presnted along the main diagonal of the big rhombus marked in (a) and is overlaid with electron density difference map $\Delta \rho=\rho_{\mathrm{gr} / \mathrm{Pd} / \mathrm{Ir}}-\left(\rho_{\mathrm{gr}}+\rho_{\mathrm{Pd} / \mathrm{Ir}}\right) . \Delta \rho$ is colour coded as red $\left(+3.5 e / \mathrm{nm}^{3}\right)-$ green (0) - blue $\left(-3.5 e / \mathrm{nm}^{3}\right)$. (c-e) Spin-resolved band structures of $\mathrm{gr} / \mathrm{Ir}(111), \mathrm{gr} / \mathrm{Pd} / \operatorname{Ir}(111)$, and $\mathrm{Pd} / \operatorname{Ir}(111)$, respectively, obtained after unfolding procedure and presented around the $K$ point of the $(1 \times 1)$ graphene-derived Brillouin zone $(\mathrm{c}, \mathrm{d})$ and $\mathrm{Pd} / \mathrm{Ir}(111)$-derived Brillouin zone (e), respectively. The $\pi$ and $\pi^{*}$ graphene bands are indicated. 
where a weakly corrugated $\left(\Delta_{\mathrm{gr}}=0.322 \AA\right)$ graphene layer is located at the mean distance of $d=3.391 \AA$ from the metallic substrate, 20 for the $\mathrm{gr} / \mathrm{Pd} / \operatorname{Ir}(111)$ intercalated system a graphene layer is highly corrugated between $2.363 \AA$ and $3.094 \AA$ with a strongly reduced mean distance between graphene and metallic support of $2.862 \AA$. Such substantial reduction of the gr-metal distance in the intercalated system leads to the modification of the electronic structure of graphene that is reflected in the difference electron density map which is shown in panel (b). Here we can notice the change of the doping sign for graphene ( $n$-doping) compared to the one for $\operatorname{gr} / \operatorname{Ir}(111)$ ( $p$-doping). $\stackrel{29}{2}$ Also the hybridization between graphene $\pi$ and $\mathrm{Pd} 4 d_{z^{2}}$ states is clearly visible at the HCP high-symmetry position of the gr $/ \mathrm{Pd} / \operatorname{Ir}(111)$ interface. Such modifications in the crystallographic structure of graphene on $\operatorname{Ir}(111)$ upon Pd-layer intercalation together with the strong induced spin-orbit interaction in Pd might lead to the drastic changes in the spin configuration in graphene as shown below.

Figure 1 (c-e) summarizes the change of the graphene electronic structure during intercalation of $1 \mathrm{MLPd}$ in $\mathrm{gr} / \operatorname{Ir}(111$ ) (all presented spin-resolved results are obtained in DFT calculations with spin-orbit interaction taken into account). According to the present results, graphene in $\operatorname{gr} / \operatorname{Ir}(111)$ is weakly $p$-doped and the sizeable band-gap is opened for the valence band states directly above $E_{F}$ as a result of hybridization of the graphene $\pi$ and Ir $5 d_{z^{2}}$ valence band states. Due to the relatively large average distance between graphene and $\operatorname{Ir}(111)$ the induced spin-orbit interaction for the graphene $\pi$ electrons is weak that leads to the small spin-splitting of the $\pi$ band in the vicinity of $E_{F}$ of only approximately $20 \mathrm{meV} .20$

The intercalation of $\mathrm{Pd}$ in $\mathrm{gr} / \mathrm{Ir}(111)$ complicates the electronic structure of the resulting system (Fig. 1 (d) and Fig. S1 in Supporting Informations); however, the dispersion of the graphene $\pi$ bands in the vicinity of the $K$ point can still be clearly recongnized allowing the analysis of the spin topology of the valence band states. As can be seen, graphene is $n$-doped in $\mathrm{gr} / \mathrm{Pd} / \operatorname{Ir}(111)$ thus its doping is reversed compared to the one of the parent 
$\operatorname{gr} / \operatorname{Ir}(111)$ system. The interesting feature of graphene in the formed intercalation system is the evolution of band gaps in the electronic spectrum of graphene. First of all, the large band gap for graphene $\pi$ states in gr/Ir(111), which is located directly above $E_{F}$, is closed, meaning that the intercalated Pd layer blocks the effective hybridization of the valence band states of graphene and Ir. Secondly, the "huge" band gap of $600 \mathrm{meV}$ is opened for the graphene $\pi$ states directly at the $K$ point. Also the lower branch of the graphene $\pi$ band becomes strongly spin-polarized demonstrating the relatively large $k$-dependent energy splitting of $50 \mathrm{meV}$ at the $K$ point and $75 \mathrm{meV}$ at $k_{\Gamma \mathrm{K}}-k_{\|}=0.1 \AA^{-1}$.

The origin of the observed effects can be understood from the analysis of the electronic structure of the $\mathrm{gr} / \mathrm{Pd} / \operatorname{Ir}(111)$ and $\mathrm{Pd} / \operatorname{Ir}(111)$ systems unfolded on the Brillouin zone corresponding to the metallic substrate's lattice (Fig. 1 (e) and Fig. S2 in the Supporting Informations). One can see that the band gap in the electronic spectrum of the graphene $\pi$ states appears as a result of the "avoid crossing" mechanism involving the $\mathrm{Pd} 4 d_{z^{2}}$ states. In its turn the respective Pd band is a simply quantum well state (QWS) which is formed in the Pd layer sandwiched between a graphene layer and the $\operatorname{Ir}(111)$ substrate. This state can be also recognised in the band structure of the intermediate system - $\mathrm{Pd} / \operatorname{Ir}(111)$ - where it appears in the energy- $k$-vector window of the electronic structure of the $\operatorname{Ir}(111)$ slab which is located around the $K$-point of the respective substrate-related Brillouin zone (Fig. 1(d)). ${ }^{29}$ As can be also seen this state is strongly spin-split due to the large spin-orbit interaction in Pd. The respective interaction between graphene and Pd layer at the relatively short distance between two layers of $2.363 \AA$ leads to the appearance of the corresponding induced spinorbit splitting of the graphene $\pi$ bands as was discussed earlier. Contrary to the previously discussed cases on the possible observation of the induced spin-orbit splitting in graphene, here two factors favour the observation of such effect in the experiment: relatively small distance between graphene and $\mathrm{Pd}$ and the hybridization of the valence band states from two layers, graphene $\pi$ and $\mathrm{Pd} 4 d_{z^{2}}$ QWSs, which appears close to the $K$-point of graphene and in 
the vicinity of $E_{F}$. Here we would like to emphasise that the present theoretical predictions are made on the basis of the DFT calculations for fully-relaxed geometry of the considered system without any structural simplification and artificial reduction of the distances between graphene and metal.

The predicted effects on the induced spin-orbit splitting of the graphene $\pi$ states are confirmed in our ARPES experiments. As was described earlier, the gr/Pd/Ir(111) system was prepared via intercalation of thin Pd layer. Since the high quality growth of well aligned graphene requires typically high temperatures at which single atomic layers on metals are normally not stable ${ }^{30}$ we proceeded by intercalation to reach the desired system. To promote Pd intercalation, the graphene layer has been grown on $\operatorname{Ir}(111)$ by temperature programmed growth (TPG) up to a coverage of $85 \%$. With such a procedure the $\operatorname{Ir}(111)$ surface is covered by a high quality percolating graphene layer in which a high density of nanometric holes are left. ${ }^{31}$ The resulting graphene layer is well aligned with the substrate with the expected moiré

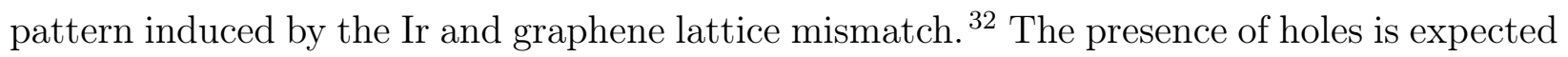
to facilitate intercalation of external species from the graphene flake edges. $\frac{33}{35}$ Fig. 2 (a,b) shows STM images of the sample after intercalation of $0.4 \mathrm{ML}$ and $0.9 \mathrm{ML}$ of Pd. It can be immediately seen that the apparent corrugation of the moiré pattern changes from about $0.1 \AA$ for $\operatorname{gr} / \operatorname{Ir}(111)$ to $0.5 \AA$ in the case of $\operatorname{gr} / \mathrm{Pd} / \operatorname{Ir}(111)$ (Fig. 2 (c)). After the deposition of a complete layer of $\mathrm{Pd}$ the system recovers a homogenous corrugation of about $0.5 \AA$ (compared to the theoretical value of $0.73 \AA$ obtained in our DFT calculations). Since such a corrugation has been seen to be independent on the tunneling parameters (tunneling current and bias voltage) we expect the real corrugation value to be not very different from the one measured by STM. $\frac{36 \mid 37}{30 r e o v e r, ~ a c c o r d i n g ~ t o ~ o u r ~ c a l c u l a t i o n s, ~ t h e ~ g r a p h e n e-P d ~ i n t e r a c t i o n ~}$ $(125 \mathrm{meV} / \mathrm{C}$-atom $)$ is stronger than the graphene-Ir one $(79 \mathrm{meV} / \mathrm{C}$-atom $)$ ensuring that the enhanced amplitude of the moire pattern is due to the $\mathrm{Pd}$ intercalation, which grows pseudomorphic on the Ir substrate. $\frac{38}{40}$ Several nanometric wrinkles develop on the graphene 
a)

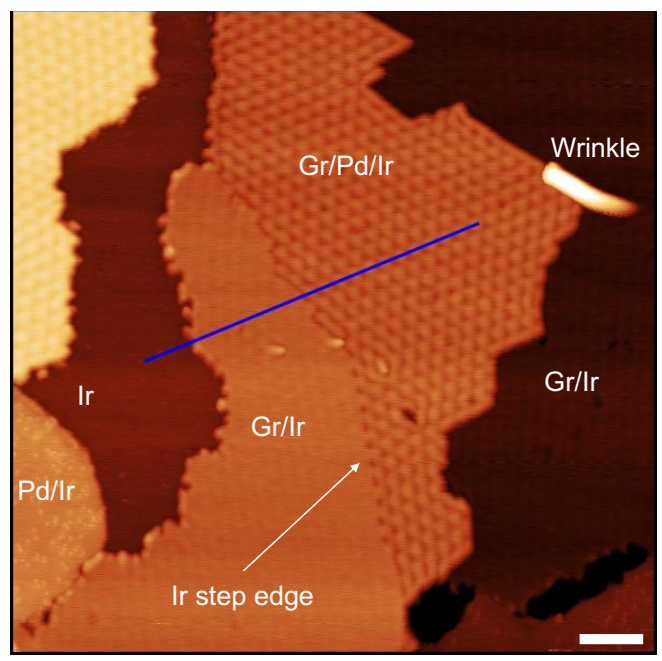

b)

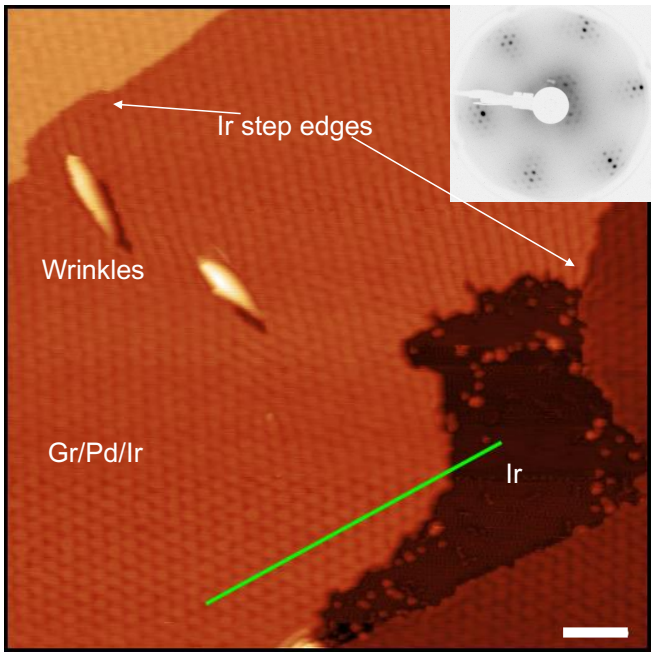

c)

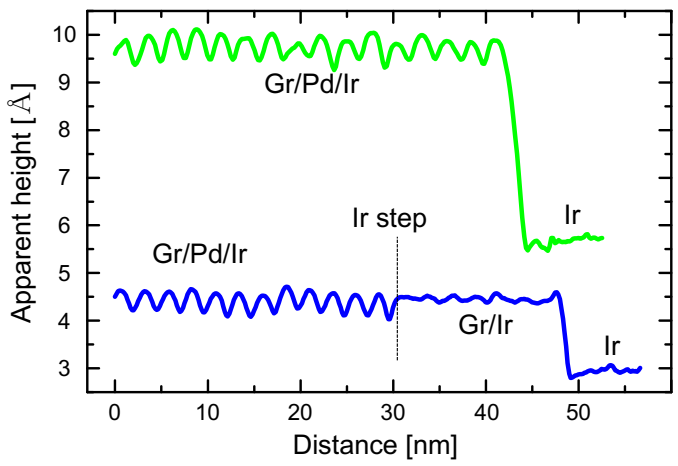

Figure 2: (a)-(b) STM images (white scalebar $10 \mathrm{~nm}$ ) of the Pd intercalated system after $0.4 \mathrm{ML}$ and $0.9 \mathrm{ML}$ respectively. Ir step edges, graphene wrinkles and the different regions of the images are indicated $\left(U_{T}=1 \mathrm{~V}, I_{T}=1 \mathrm{nA}\right)$. Inset of (b) shows LEED pattern $(70 \mathrm{eV})$ after one ML Pd intercalation under graphene grown by TPG until $85 \%$ coverage. (c) Apparent height profiles taken along the blue and green lines (green shifted for clarity) shown in figure (a) and (b) respectively. 
surface. Following previous works on the intercalation of materials strongly interacting with carbon atoms, we attribute the presence of wrinkles to stress relief in the graphene layer. 355141 After one ML Pd intercalation, as expected, the LEED diffraction pattern of gr/Pd/Ir(111) results very similar to the one of pure graphene on $\operatorname{Ir}(111)$ and no variation of the in-plane lattice parameters have been detected within our resolution (inset of Fig. 2 (b)).
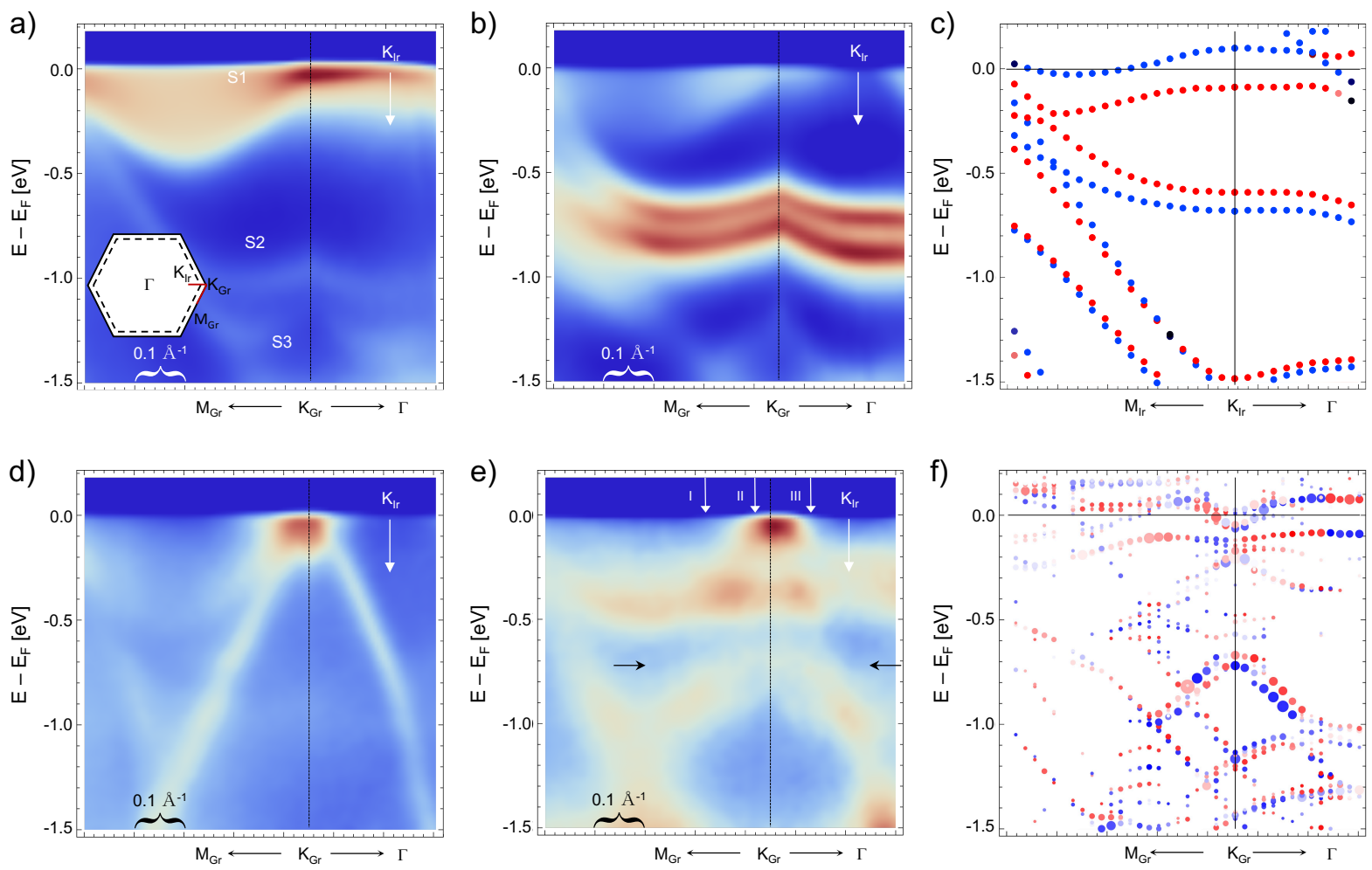

Figure 3: ARPES intensity maps along the $M_{\mathrm{gr}}-K_{\mathrm{gr}}-K_{\mathrm{Ir}}-\Gamma$ direction for (a) $\operatorname{Ir}(111)$ and (b) $\mathrm{Pd} / \operatorname{Ir}(111)$ respectively. (c) Calculated spin-resolved band structure of $\mathrm{Pd} / \operatorname{Ir}(111)$ shown in the same energy region of (a) and (b) around $K_{I r}$. (d) and (e) ARPES intensity maps along the $M_{\mathrm{gr}}-K_{\mathrm{gr}}-K_{\mathrm{Ir}}-\Gamma$ direction for gr $/ \operatorname{Ir}(111)$, and gr $/ \mathrm{Pd} / \operatorname{Ir}(111)$, respectively. (f) Calculated spin-resolved band structure of gr/Pd/Ir(111) shown in the same energy and reciprocal space regions of (d) and (e). For (d) and (e), graphene, grown by TPG up to $85 \%$ coverage, has identical band structure to a full graphene layer on $\operatorname{Ir}(111)$. Inset of (a) shows a scheme of the graphene (solid black line) and Ir (dashed black line) Brillouin zones. The relevant high symmetry points and the reciprocal space region shown are indicated.

Before addressing the graphene electronic properties we focus our attention on the elec- 
tronic structure of the $1 \mathrm{ML} P d / \operatorname{Ir}(111)$ system. With the chosen growth conditions Pd grows pseudomorphic to the Ir substrate as demonstrated by the LEED diffraction pattern in Fig. S3 of the Supporting Informations where only the six spots corresponding to the $\operatorname{Ir}(111)$ surface are visible. Since the goal of the present work is to address the electronic properties of the graphene layer, then the ARPES data are presented along the $M_{\mathrm{gr}}-K_{\mathrm{gr}}-K_{\mathrm{Ir}}-\Gamma$ direction of the Brillouin zone as depicted in the inset of Fig. 3 (a), i. e. the region where the Dirac cone of graphene is expected. Here the electronic structure of the $\operatorname{Ir}(111)$ substrate, as measured in ARPES experiment and shown in Fig. 3 (a), has conveniently a band gap and 3 surface states, indicated as $S_{1}, S_{2}$ and $S_{3} \cdot{ }^{42}$ Upon $1 \mathrm{ML}$ Pd deposition, two emission lines, which were identified in our DFT calculations as a spin-orbit split QWS, develop in the band gap (Fig. 3(b)). Those low dispersing states are situated between $E-E_{F}=-0.5 \mathrm{eV}$ and $E-E_{F}=-1.0 \mathrm{eV}$ in the measurement region. As expected their interaction with the Ir surface states is quite strong producing a shift of few hundreds of meV for the $S_{3}$ state. The energy splitting between two components of the observed Pd-induced QWS goes from $170 \mathrm{meV}$ at the gr $K$ point to $120 \mathrm{meV}$ at the Ir $K$ point. For comparison, Fig. 3 (c) show the spin resolved DFT calculations of $1 \mathrm{ML}$ Pd/Ir(111) system in the reciprocal space region close to that of Fig. 3 (b), around $K_{I r}$, showing the excellent agreement between the experimental and theoretical results.

The results of the ARPES measurements for gr/Ir(111) and gr/Pd/Ir(111) are compared in Fig. 3(d) and (e) along the same direction highlighted in Fig. 3 (a). The band dispersion of our TPG graphene is identical, within our resolution, to the one of a full graphene layer on $\operatorname{Ir}(111) \cdot 4244$ The high spectral intensity close to the Dirac point is due to the superposition of the Dirac cone and the $S_{1}$ Ir surface state, which spectral weight is strongly enhanced in our measurements conditions (see Fig. 3 (a)).

Upon Pd intercalation, strong modifications of the system band structure with respect to the one of graphene on $\operatorname{Ir}(111)$ and $\operatorname{Pd}$ on $\operatorname{Ir}(111)$ are immediately evident. The band 
dispersion along the $M_{\mathrm{gr}}-K_{\mathrm{gr}}-K_{\mathrm{Ir}}-\Gamma$ direction of the Brillouin zone can be analyzed considering three energy regions in which several electronic states are present. The first one of $E-E_{F}=-1.5 \ldots-0.7 \mathrm{eV}$ presents hole like dispersing states with apex at the $K_{\mathrm{gr}}$ point at $E-E_{F}=-0.7 \mathrm{eV}$. The second one of $E-E_{F}=-0.5 \ldots-0.3 \mathrm{eV}$ shows a rather flat sets of states that traverse the reciprocal space, and finally a high electronic density can be found at the Fermi level located at $K_{\mathrm{gr}}$. It is evident that in the first two energy regions the electronic states present a rather complex band dispersion. Comparing the states from the first region with the results of our DFT calculations presented in Fig. 1 (a zoom in the same energy and reciprocal space window of the experimental results is shown in Fig. 3(f) for comparison) we can identify these states as spin-orbit split graphene-derived $\pi$ bands in $\mathrm{gr} / \mathrm{Pd} / \operatorname{Ir}(111)$. For this purpose, we show three electron distribution curves (EDCs) in Fig. 4 (a) (black dashed lines) corresponding to the momentum highlighted by the three white arrows in Fig. 3 (e). From the comparison of these curves it is evident that the graphene-derived $\pi$ band has indeed two components. Although the same analysis is more difficult for the low dispersing state in the second energy region, we can identify a two-states structure along the $M_{\mathrm{gr}}-K_{\mathrm{gr}}$ far from the graphene $K$ point (see profile I in Fig. 4(a)). In order to quantitatively evaluate the presence of a doublet of electronic states we fit the EDCs, in the momentum region highlighted by the black arrows in Fig. 3 (e), with a double Gaussian function summed with a linear background. Examples of such fitting procedure are given in Fig. 4(a) where the green curve represent the fitted function while red and blue curves correspond to its two components. The resulting Gaussian peak positions are shown in Fig. 4(b). For sake of simplicity only the error bars corresponding to the three profiles shown in Fig. 4(a), are presented. For the graphene-derived $\pi$ band we can detect two separated electronic contributions along the $M_{\mathrm{gr}}-K_{\mathrm{gr}}-K_{\mathrm{Ir}}-\Gamma$ in a region of about $0.3^{-1}$ centered at the $K_{\mathrm{gr}}$ point. This energy separation reaches more than $200 \mathrm{meV}$ and it is strongly reduced at the graphene $K$ point. The situation of the low dispersing state is 
rather more complex. While in the majority of the considered reciprocal space points the two components lies within the error bars, we can detect a clear $200 \mathrm{meV}$ energy separation along the $M_{\mathrm{gr}}-K_{\mathrm{gr}}$ direction at about $0.15^{-1}$ from the $K_{\mathrm{gr}}$ point.

The comparison between DFT simulations and ARPES experiments allow us to point out two fundamental properties of the gr/Pd/Ir(111) system. Firstly, as was discussed earlier, the graphene $\pi$ band possess spin-orbit splitting with a momentum dependent energy separation. This is a crucial aspect which allows us to identify induced spin-orbit coupling by direct hybridization, as the leading mechanism for the transfer of the strong spin-orbit interaction from $\mathrm{Pd}$ to carbon atoms. Secondly, the high electronic density located at $K_{\text {gr }}$ at the Fermi level (Fig. $3(\mathrm{e})$ ) corresponds to the $\pi^{*}$ graphene-derived state showing therefore the opening of a large bandgap, of about $600 \mathrm{meV}$, in the graphene band structure.

In conclusion, we demonstrated that a strong spin-orbit splitting can be induced in graphene $\pi$ state by its interaction with $1 \mathrm{ML} \mathrm{Pd}$ on $\operatorname{Ir}(111)$. After intercalation of $\mathrm{Pd}$ in gr/Ir(111), two fully spin polarized Pd-derived QWS develop in the momentum-energy region of the graphene Dirac cone. As shown in our DFT calculations this spin splitting of the $\mathrm{Pd}$ QWS is a result of the strong induced spin-orbit interaction at the $\mathrm{Pd} / \mathrm{Ir}(111)$ interface. The resulting band structure of $\mathrm{gr} / \mathrm{Pd} / \operatorname{Ir}(111)$ demonstrates that graphene is strongly $n$ doped and the energy gap of $600 \mathrm{meV}$ appears in the dispersion of the $\pi$ band at the $K$ point. The graphene Dirac cone strongly hybridizes with the spin-split Pd-derived QWS in the $\mathrm{gr} / \mathrm{Pd} / \operatorname{Ir}(111)$ intercalated system that leads to the significant spin-orbit interaction transfer to the graphene $\pi$ states. As a result, the band dispersion of these states around the $K$ point and in the vicinity of the Fermi level demonstrates a momentum dependent spin-splitting reaching values higher than $200 \mathrm{meV}$. The strong hybridization is confirmed by DFT calculations which reproduces the system band structure and spin topology of the electronic states of graphene, considering a realistic average $\mathrm{Pd}-\mathrm{C}$ distance. These results demonstrate that the proposed approach on the tailoring of the dimensionality of heavy 

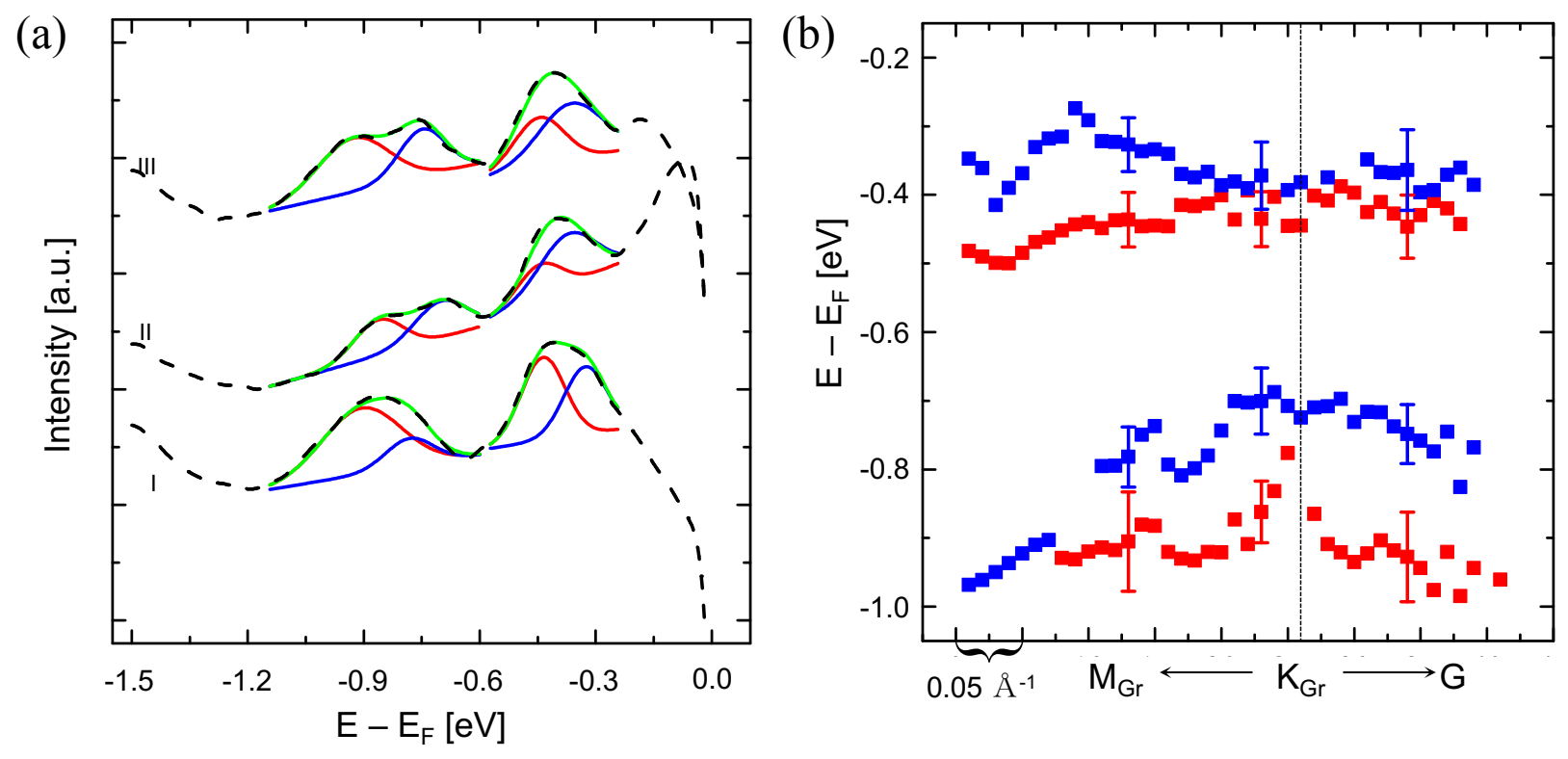

Figure 4: (a) Electron distribution curves at the wave vectors indicated by the white arrows in Fig. 3 (e). The dashed lines correspond to the experimental data, while the green, red and blue lines correspond to the fitted function, and its two gaussian components, respectively. (b) Positions of the gaussian centers in the momentum region enclosed by the two black arrows in Fig. 3 (e). The error bars are given only for the three fits in (a). 
materials interfaced with a graphene layer might lead to the induced giant spin-orbit splitting of the graphene valence band states. In fact, spin-polarized quantum well states exist in several other systems ${ }^{45}$ a8 and might exist for other single atomic layers on surfaces. If graphene hybridizes with such QWSs, the induced spin-polarization in the graphene valence band could be even stronger than what reported here. The same approach can be also extended to other two dimensional materials which, once interfaced with systems owning spin-polarized quantum well states, could exhibit a wide range of novel quantum mechanical properties.

\section{Contribution}

S.V. proposed the study with the help of E.V., Y.D., M.F., S.P. and D.R.. T.V. made the ARPES measurements and their analysis with the help of S.V. and S.P.. T.V. made the STM measurements with the help of S.S., M.F. and S.V.. E.V., K.W., and B.P. performed DFT calculations and analyzed the results. S.V., E.V. and Y.D. wrote the manuscript with input of all authors. All authors discussed the results and contributed to their interpretation.

\section{Acknowledgement}

S.P. and D.R. acknowledge the region Ile de France for the support of the Nanospecs project in the framework of the C'NANO Ile de France founding agency. E.V. and Y.D. thank the support by the Ministry of Education and Science of Russian Federation within the framework of the State Assignment for Research, Grants no. 4.6759.2017/8.9. The computing facilities of the Freie Universität Berlin (ZEDAT) and the North-German Supercomputing Alliance (HLRN) are acknowledged for computer time. K.W. thanks the China Scholarship council (CSC) for financial support via a $\mathrm{PhD}$ fellowship 


\section{References}

(1) Pesin, D.; MacDonald, A. H. Spintronics and pseudospintronics in graphene and topological insulators. Nat. Mater. 2012, 11, 409.

(2) Han, W.; Kawakami, R. K.; Gmitra, M.; Fabian, J. Graphene spintronics. Nat. Nanotechnol. 2014, 9, 794.

(3) Ferrari, A. C.; Bonaccorso, F.; Fal'ko, V.; Novoselov, K. S.; Roche, S.; Bøggild, P.; Borini, S.; Koppens, F. H. L.; Palermo, V.; Pugno, N. et al. Science and technology roadmap for graphene, related two-dimensional crystals, and hybrid systems. Nanoscale 2015, 7, 4598-4810.

(4) Kane, C. L.; Mele, E. J. Quantum Spin Hall Effect in Graphene. Phys. Rev. Lett. 2005, $95,226801$.

(5) Sichau, J.; Prada, M.; Anlauf, T.; Lyon, T. J.; Bosnjak, B.; Tiemann, L.; Blick, R. H. Resonance Microwave Measurements of an Intrinsic Spin-Orbit Coupling Gap in

Graphene: A Possible Indication of a Topological State. Phys. Rev. Lett. 2019, 122, 046403.

(6) Yan, W.; Sagasta, E.; Ribeiro, M.; Niimi, Y.; Hueso, L. E.; Casanova, F. Large room temperature spin-to-charge conversion signals in a few-layer graphene/Pt lateral heterostructure. Nat. Commun. 2017, 8, 661.

(7) Klinovaja, J.; Loss, D. Giant Spin-Orbit Interaction Due to Rotating Magnetic Fields in Graphene Nanoribbons. Phys. Rev. X 2013, 3, 011008.

(8) Dedkov, Y. S.; Fonin, M.; Rüdiger, U.; Laubschat, C. Rashba Effect in the Graphene/Ni(111) System. Phys. Rev. Lett. 2008, 100, 107602. 
(9) Weser, M.; Rehder, Y.; Horn, K.; Sicot, M.; Fonin, M.; Preobrajenski, A. B.; Voloshina, E. N.; Goering, E.; Dedkov, Y. S. Induced magnetism of carbon atoms at the graphene/Ni(111) interface. Appl. Phys. Lett. 2010, 96, 012504.

(10) Dedkov, Y. S.; Fonin, M. Electronic and magnetic properties of the graphene-ferromagnet interface. New J. Phys. 2010, 12, 125004.

(11) Usachov, D.; Fedorov, A.; Otrokov, M. M.; Chikina, A.; Vilkov, O.; Petukhov, A.; Rybkin, A. G.; Koroteev, Y. M.; Chulkov, E. V.; Adamchuk, V. K. et al. Observation of Single-Spin Dirac Fermions at the Graphene/Ferromagnet Interface. Nano Lett. 2015, $15,2396-2401$.

(12) Marchenko, D.; Varykhalov, A.; Sánchez-Barriga, J.; Rader, O.; Carbone, C.; Bihlmayer, G. Highly spin-polarized Dirac fermions at the graphene/Co interface. Phys. Rev. B 2015, 91, 235431.

(13) Marchenko, D.; Varykhalov, A.; Scholz, M. R.; Bihlmayer, G.; Rashba, E. I.; Rybkin, A.; Shikin, A. M.; Rader, O. Giant Rashba splitting in graphene due to hybridization with gold. Nat. Commun. 2012, 3, 1232.

(14) Marchenko, D.; Sánchez-Barriga, J.; Scholz, M. R.; Rader, O.; Varykhalov, A. Spin splitting of Dirac fermions in aligned and rotated graphene on $\operatorname{Ir}(111)$. Phys. Rev. B 2013, 87, 115426.

(15) Calleja, F.; Ochoa, H.; Garnica, M.; Barja, S.; Navarro, J. J.; Black, A.; Otrokov, M. M.; Chulkov, E. V.; Arnau, A.; Vázquez de Parga, A. L. et al. Spatial variation of a giant spin-orbit effect induces electron confinement in graphene on $\mathrm{Pb}$ islands. Nat. Phys. 2014, 11, 43 .

(16) Klimovskikh, I. I.; Otrokov, M. M.; Voroshnin, V. Y.; Sostina, D.; Petaccia, L.; Di Santo, G.; Thakur, S.; Chulkov, E. V.; Shikin, A. M. Spin-Orbit Coupling Induced 
Gap in Graphene on $\mathrm{Pt}(111)$ with Intercalated Pb Monolayer. ACS Nano 2017, 11, 368.

(17) Klimovskikh, I. I.; Vilkov, O.; Usachov, D. Y.; Rybkin, A. G.; Tsirkin, S. S.; Filianina, M. V.; Bokai, K.; Chulkov, E. V.; Shikin, A. M. Variation of the character of spin-orbit interaction by $\mathrm{Pt}$ intercalation underneath graphene on $\operatorname{Ir}(111)$. Phys. Rev. B 2015, 92, 165402.

(18) Otrokov, M. M.; Klimovskikh, I. I.; Calleja, F.; Shikin, A. M.; Vilkov, O.; Rybkin, A. G.; Estyunin, D.; Muff, S.; Dil, J. H.; de Parga, A. L. V. et al. Evidence of large spin-orbit coupling effects in quasi-free-standing graphene on $\mathrm{Pb} / \operatorname{Ir}(111)$. 2D Mater. 2018, 5, 035029 .

(19) Rybkin, A. G.; Rybkina, A. A.; Otrokov, M. M.; Vilkov, O. Y.; Klimovskikh, I. I.; Petukhov, A. E.; Filianina, M. V.; Voroshnin, V. Y.; Rusinov, I. P.; Ernst, A. et al. Magneto-Spin-Orbit Graphene: Interplay between Exchange and Spin-Orbit Couplings. Nano Lett. 2018, 18, 1564.

(20) Voloshina, E.; Dedkov, Y. Realistic Large-Scale Modeling of Rashba and Induced SpinOrbit Effects in Graphene/High-Z-Metal Systems. Adv. Theory and Simul. 2018, 1, 1800063.

(21) Brede, J.; Slawinska, J.; Abadia, M.; Rogero, C.; Ortega, J. E.; Piquero-Zulaica, I.; Lobo-Checa, J.; Arnau, A.; Cerda, J. I. Tuning the Graphene on $\operatorname{Ir}(111)$ adsorption regime by Fe/Ir surface- alloying. 2D Mater. 2016, 4, 015016.

(22) Enderlein, C.; Kim, Y. S.; Bostwick, A.; Rotenberg, E.; Horn, K. The formation of an energy gap in graphene on ruthenium by controlling the interface. New J. Phys. 2010, 12, 033014 . 
(23) Starodub, E.; Bostwick, A.; Moreschini, L.; Nie, S.; Gabaly, F. E.; McCarty, K. F.; Rotenberg, E. In-plane orientation effects on the electronic structure, stability, and Raman scattering of monolayer graphene on $\operatorname{Ir}(111)$. Phys. Rev. B 2011, 83, 125428.

(24) Wofford, J. M.; Starodub, E.; Walter, A. L.; Nie, S.; Bostwick, A.; Bartelt, N. C.; Thürmer, K.; Rotenberg, E.; McCarty, K. F.; Dubon, O. D. Extraordinary epitaxial alignment of graphene islands on $\mathrm{Au}(111)$. New J. Phys. 2012, 14, 053008.

(25) Leicht, P.; Tesch, J.; Bouvron, S.; Blumenschein, F.; Erler, P.; Gragnaniello, L.; Fonin, M. Rashba splitting of graphene-covered $\mathrm{Au}(111)$ revealed by quasiparticle interference mapping. Phys. Rev. B 2014, 90, 241406.

(26) Tesch, J.; Leicht, P.; Blumenschein, F.; Gragnaniello, L.; Fonin, M.; Marsoner Steinkasserer, L. E.; Paulus, B.; Voloshina, E.; Dedkov, Y. Structural and electronic properties of graphene nanoflakes on $\mathrm{Au}(111)$ and $\mathrm{Ag}(111)$. Sci. Rep. 2016, 6, 23439 .

(27) Tesch, J.; Voloshina, E.; Jubitz, M.; Dedkov, Y.; Fonin, M. Local electronic properties of the graphene-protected giant Rashba-split $\mathrm{BiAg}_{2}$ surface. Phys. Rev. B 2017, 95, 155428.

(28) Vanin, M.; Mortensen, J. J.; Kelkkanen, A. K.; Garcia-Lastra, J. M.; Thygesen, K. S.; Jacobsen, K. W. Graphene on metals: A van der Waals density functional study. Phys. Rev. B 2010, 81, 081408 .

(29) Pletikosić, I.; Kralj, M.; Šokčević, D.; Brako, R.; Lazić, P.; Pervan, P. Photoemission and density functional theory study of $\operatorname{Ir}(111)$ energy band gap mapping. J. Phys.: Condens. Matter 2010, 22, 135006.

(30) Coraux, J.; N’Diaye, A. T.; Rougemaille, N.; Vo-Van, C.; Kimouche, A.; Yang, H.X.; Chshiev, M.; Bendiab, N.; Fruchart, O.; Schmid, A. K. Air-Protected Epitaxial 
Graphene/Ferromagnet Hybrids Prepared by Chemical Vapor Deposition and Intercalation. J. Phys. Chem. Lett. 2012, 3, 2059.

(31) Coraux, J.; N’Diaye, A. T.; Engler, M.; Busse, C.; Wall, D.; Buckanie, N.; zu Heringdorf, F.-J. M.; van Gastel, R.; Poelsema, B.; Michely, T. Growth of graphene on Ir(111). New J. Phys. 2009, 11, 023006.

(32) Coraux, J.; N’Diaye, A. T.; Busse, C.; Michely, T. Structural Coherency of Graphene on $\operatorname{Ir}(111)$. Nano Lett. 2008, 8, 565.

(33) Vlaic, S.; Kimouche, A.; Coraux, J.; Santos, B.; Locatelli, A.; Rougemaille, N. Cobalt intercalation at the graphene/iridium(111) interface: Influence of rotational domains, wrinkles, and atomic steps. Appl. Phys. Lett. 2014, 104, 101602.

(34) Vlaic, S.; Rougemaille, N.; Kimouche, A.; Burgos, B. S.; Locatelli, A.; Coraux, J. Intercalating cobalt between graphene and iridium (111): Spatially dependent kinetics from the edges. Phys. Rev. Materials 2017, 1, 053406.

(35) Vlaic, S.; Rougemaille, N.; Artaud, A.; Renard, V.; Huder, L.; Rouvière, J.-L.; Kimouche, A.; Santos, B.; Locatelli, A.; Guisset, V. et al. Graphene as a Mechanically Active, Deformable Two-Dimensional Surfactant. J. Phys. Chem. Lett. 2018, 9, 2523.

(36) Voloshina, E. N.; Fertitta, E.; Garhofer, A.; Mittendorfer, F.; Fonin, M.; Thissen, A.; Dedkov, Y. S. Electronic structure and imaging contrast of graphene moiré on metals. Sci. Rep. 2013, 3, 1072.

(37) Dedkov, Y.; Voloshina, E. Multichannel scanning probe microscopy and spectroscopy of graphene moiré structures. Phys. Chem. Chem. Phys. 2014, 16, 3894-3908.

(38) Sicot, M.; Leicht, P.; Zusan, A.; Bouvron, S.; Zander, O.; Weser, M.; Dedkov, Y. S.; 
Horn, K.; Fonin, M. Size-Selected Epitaxial Nanoislands Underneath Graphene Moiré on $\mathrm{Rh}(111)$. ACS Nano 2012, 6, 151-158.

(39) Decker, R.; Brede, J.; Atodiresei, N.; Caciuc, V.; Blügel, S.; Wiesendanger, R. Atomicscale magnetism of cobalt-intercalated graphene. Phys. Rev. B 2013, 8\%, 041403.

(40) Li, G.; Zhang, Y.-Y.; Guo, H.; Huang, L.; Lu, H.; Lin, X.; Wang, Y.-L.; Du, S.; Gao, H.-J. Epitaxial growth and physical properties of 2D materials beyond graphene: from monatomic materials to binary compounds. Chem. Soc. Rev. 2018, 47, 6073-6100.

(41) N’Diaye, A. T.; van Gastel, R.; Martínez-Galera, A.; Coraux, J.; Hattab, H.; Wall, D.; Meyer zu Heringdorf, F.; Horn-von Hoegen, M.; Gomez-Rodriguez, J. M.; Poelsema, B. et al. In situ observation of stress relaxation in epitaxial graphene. New J. Phys. 2009, 11.

(42) Pletikosić, I.; Kralj, M.; Pervan, P.; Brako, R.; Coraux, J.; N’Diaye, A. T.; Busse, C.; Michely, T. Dirac Cones and Minigaps for Graphene on $\operatorname{Ir}(111)$. Phys. Rev. Lett. 2009, 102, 056808.

(43) Rusponi, S.; Papagno, M.; Moras, P.; Vlaic, S.; Etzkorn, M.; Sheverdyaeva, P. M.; Pacilé, D.; Brune, H.; Carbone, C. Highly Anisotropic Dirac Cones in Epitaxial Graphene Modulated by an Island Superlattice. Phys. Rev. Lett. 2010, 105, 246803.

(44) Vita, H.; Böttcher, S.; Horn, K.; Voloshina, E. N.; Ovcharenko, R. E.; Kampen, T.; Thissen, A.; Dedkov, Y. S. Understanding the origin of band gap formation in graphene on metals: graphene on $\mathrm{Cu} / \operatorname{Ir}(111)$. Sci. Rep. 2014, 4, 5704 .

(45) Altmann, K. N.; O’Brien, W.; Seo, D. J.; Himpsel, F. J.; Ortega, J. E.; Naermann, A.; Segovia, P.; Mascaraque, A.; Michel, E. G. Spin-polarized quantum well states. J. Electron Spectrosc. 1999, 101, 367. 
(46) He, K.; Hirahara, T.; Okuda, T.; Hasegawa, S.; Kakizaki, A.; Matsuda, T. Spin Polarization of Quantum Well States in Ag Films Induced by the Rashba Effect at the Surface. Phys. Rev. Lett. 2008, 101, 107604.

(47) Yee, M. M.; Zhu, Z.-H.; Soumyanarayanan, A.; He, Y.; Song, C.-L.; Pomjakushina, E.; Salman, Z.; Kanigel, A.; Segawa, K.; Ando, Y. et al. Spin-polarized quantum well states on $\mathrm{Bi}_{2-x} \mathrm{Fe}_{x} \mathrm{Se}_{3}$. Phys. Rev. B 2015, 91, 161306.

(48) Kormondy, K. J.; Gao, L.; Li, X.; Lu, S.; Posadas, A. B.; Shen, S.; Tsoi, M.; McCartney, M. R.; Smith, D. J.; Zhou, J. et al. Large positive linear magnetoresistance in the two-dimensional t2gelectron gas at the EuO/SrTiO3 interface. Sci. Rep. 2018, 8, 7721. 